\title{
Cosmological implications of a supersymmetric extension of the Brans-Dicke theory
}

\author{
Riccardo Catena \\ Deutsches Elektronen-Syncrotron (DESY), 22603 Hamburg, Germany
}

\begin{abstract}
In the Brans-Dicke theory the Planck mass is replaced by a dynamical scalar field. We consider here the supersymmetric analogous of this mechanism replacing in the supergravity Lagrangian the Planck mass with a chiral superfield. This analysis is motivated by the research of possible connections between supersymmetric Dark Matter scenarios and Dark Energy models based on Brans-Dicke-like theories. We find that, contrary to the original Brans-Dicke theory, in its supersymmetric analogous the gravitational sector does not couple to the matter sector in a universal metric way. As a result, violations of the weak equivalence principle could be present in such a scenario.
\end{abstract}

Keywords: Scalar-Tensor theories, Cosmology, Supersymmetry

PACS: $04.50 .+\mathrm{h}, 04.65 .+\mathrm{e}$

\section{INTRODUCTION}

In the Brans-Dicke approach to the gravitational interaction, the Planck mass is replaced in the Lagrangian by a dynamical scalar field $\varphi$ [1]. Even though, as a theory of gravity, the original Brans-Dicke theory is at present very constrained by solar system measurements [2], its modern versions, namely Scalar-Tensor (ST) theories [3] - where a non trivial potential is associated to $\varphi$ - can still pass all cosmological and astrophysical bounds leading at the same time to interesting and testable predictions [4].

Like in General Relativity, in ST theories matter couples to gravity in a universal metric way i.e. all matter fields feel the gravitational interaction only through one and the same metric. Because of this property, ST theories are by construction protected by any violation of the weak equivalence principle and even ultra-light degrees of freedom mediating long range forces are not in this framework phenomenologically dangerous [5]. Such a feature makes ST theories interesting arenas for dynamical Dark Energy model building[6, 7].

However, a realistic cosmological scenario can be only achieved when Dark Matter is consistently included to the picture [8]. Among different theories providing suitable Dark Matter candidates, Supersymmetry is certainly one of the most remarkable [9].

The interesting possibility to relate a ST interpretation of Dark Energy to a supersymmetric description of Dark Matter leads to study supersymmetric extensions of ST theories. This is the topic of the present work.

We consider here the supersymmetric analogous of the BD idea: we replace in the supergravity Lagrangian the Planck mass with a chiral superfield, the "Planck superfield". Such a replacement defines the "natural" supersymmetric extension of the BD 
theory. Let us refer to it as the Minimal Supersymmetric Brans-Dicke theory (MSBD) to distinguish it from other possible approaches. We find that, contrary to the original BD theory, in the MSBD the gravitational sector does not couple to the matter sector in a universal metric way. As a result, possible violations of the weak equivalence principle could make the minimal supersymmetric extension of the BD idea phenomenologically inconsistent.

The plan of this work is as follows. In the next section we review the BD model and the concept of universal metric coupling. The third section is devoted to the MSBD theory; we will specially underline the differences between its phenomenology and the one of the original BD theory. The results are finally discussed in the Conclusions. For notation we refer in the following to $[10,11]$.

\section{THE BRANS-DICKE THEORY AND THE UNIVERSAL METRIC COUPLING}

In General Relativity the coupling between gravity and matter is described by the following Lagrangian

$$
\mathscr{L}_{E H}=-\frac{1}{2} e M_{P l}^{2} \mathscr{R}+\mathscr{L}_{M}\left[e_{m}^{a}, \Psi\right],
$$

where $e \equiv \operatorname{det}\left(e_{m}^{a}\right), \mathscr{R}$ is the Ricci scalar and $\Psi$ symbolically represents all matter fields involved in the theory. In the BD approach to the gravitational interaction the Planck mass appearing in eq. (1) becomes dynamical by means of the substitution

$$
M_{P l}^{2} \Longrightarrow \varphi^{2}\left(y^{m}\right),
$$

where $\varphi\left(y^{m}\right)$ is a real scalar field. As a consequence eq. (1) is replaced by

$$
\begin{aligned}
\mathscr{L}_{B D} & =\mathscr{L}_{\varphi}\left[e_{m}^{a}, \varphi\right]+\mathscr{L}_{M}\left[e_{m}^{a}, \Psi\right] \\
& =-\frac{1}{2} e\left(\varphi^{2} \mathscr{R}+\omega \partial_{m} \varphi \partial^{m} \varphi\right)+\mathscr{L}_{M}\left[e_{m}^{a}, \Psi\right],
\end{aligned}
$$

where the factor $\omega$ that multiplies the kinetic term of $\varphi$ has to be tuned to fit the postnewtonian bounds [2]. Eq. (3) gives the so called "Jordan frame" formulation of the theory. In this frame the BD scalar does not appear in the matter Lagrangian and particle physics is just the standard one. The theory can be formulated in other frames related to the Jordan one by a Weyl rescaling of the vielbein such as $e_{m}^{a} \rightarrow e_{m}^{a} e^{l(\varphi)}$, where $l(\varphi)$ is some $\varphi$-dependent function. In these alternative formulations the matter Lagrangian acquires an explicit functional dependence from $\varphi$, i.e. $\mathscr{L}_{M}=\mathscr{L}_{M}\left[e_{m}^{a} e^{l(\varphi)}, \Psi\right]$. However, the inverse Weyl rescaling $e_{m}^{a} \rightarrow e_{m}^{a} e^{-l(\varphi)}$ always brings back the theory to its original version in which particle physics is just the standard one.

Eq. (3) shows that in the BD theory all matter fields feel the gravitational interaction through the same vielbein, the Jordan frame vielbein. For this reason such a mattergravity coupling is also called universal and metric. This is a non trivial property and has very important phenomenological implications. It can be shown, for instance, that in a theory where matter couples to gravity in a universal metric way the weak equivalence principle is satisfied by construction [5]. 


\section{THE MINIMAL SUPERSYMMETRIC BRANS-DICKE THEORY}

Eq. (2) gives a prescription to construct the BD Lagrangian starting from the EinsteinHilbert one. In this section we apply an analogous prescription to the supergravity Lagrangian

$$
\mathscr{L}_{s g}=-3 M_{P l}^{2} \int d^{2} \theta 2 \mathscr{E} R+\mathscr{L}_{M}[H, \Psi]+\text { h.c. },
$$

where $H$ is the supergravity multiplet, $\mathscr{E}$ is the chiral density and $R$ represents the curvature superfield, defined as the covariant derivative of the spin connection.

Let us start introducing a chiral superfield $\Phi$ with components given by the power series expansion $\Phi\left(y^{m}, \theta_{\alpha}\right)=A\left(y^{m}\right)+\sqrt{2} \theta^{\alpha} \chi_{\alpha}\left(y^{m}\right)+\theta^{\alpha} \theta_{\alpha} F\left(y^{m}\right)$, where $A\left(y^{m}\right)$ and $F\left(y^{m}\right)$ are complex scalars and $\chi_{\alpha}\left(y^{m}\right)$ a Weyl spinor. We will call $\Phi$ the Planck superfield. This dynamical object allows the natural supersymmetric extension of the substitution (2)

$$
M_{P l}^{2} \Longrightarrow \Phi^{2}\left(y^{m}, \theta_{\alpha}\right) \text {. }
$$

Applying the substitution (5) to eq. (4) one finds

$$
\begin{aligned}
\mathscr{L}_{M S B D} & =\mathscr{L}_{\Phi}[H, \Phi]+\mathscr{L}_{M}[H, \Psi] \\
& =-3 \int d^{2} \theta \Phi^{2} 2 \mathscr{E} R- \\
& -\frac{1}{8} \int d^{2} \theta 2 \mathscr{E}\left(\overline{\mathscr{D}}_{\dot{\alpha}} \overline{\mathscr{D}}^{\dot{\alpha}}-8 R\right) \Phi^{\dagger} \Phi+ \\
& +\mathscr{L}_{M}[H, \Psi]+\text { h.c. },
\end{aligned}
$$

where in the third line, in analogy with eq. (3), we introduced a kinetic term for $\Phi$. To be as general as possible we do not assume any particular form for $\mathscr{L}_{M}$.

Eq. (6) defines the Minimal Supersymmetric Brans Dicke theory (MSBD). Its invariance under supergravity transformations follows from the properties of chiral densities. By definitions, chiral densities transform like total derivatives in the space $\left(y^{m}, \theta_{\alpha}\right)$ and the product of a chiral density and a chiral superfield is again a chiral density [10]. Moreover, the superfields $(\overline{\mathscr{D}} \overline{\mathscr{D}}-8 R) \Phi^{\dagger} \Phi$ and $\Phi^{2}$ are chiral if $\Phi$ is chiral. This proves the invariance of the Lagrangian (6) under supergravity transformations.

Let us focus now on its phenomenology. As it was shown in [11], the component fields expansion of eq. (6) gives rise to a Lagrangian with the following structure

$$
\mathscr{L}_{M S B D}=\mathscr{L}_{\Phi}\left[e_{m}^{a}, \psi_{\alpha}^{a}, b^{a}, M, A, \chi_{\alpha}, F\right]+\mathscr{L}_{M}\left[e_{m}^{a}, \psi_{\alpha}^{a}, b^{a}, M, \Psi\right],
$$

where we introduced the gravitino $\psi_{\alpha}^{a}$ and two auxiliary fields: a vector $b^{a}$ and a scalar $M$. Eq. (7) is the supersymmetric version of eq. (3). The crucial difference between the two Lagrangians is that in the supersymmetric one $\mathscr{L}_{M}$ and $\mathscr{L}_{\Phi}$ communicate also through the auxiliary fields $b^{a}$ and $M$. This has deep phenomenological consequences when the auxiliary fields are removed by means of their equations of motion. To show this point, let us write the general solution of the equations of motion for $M$ and $b^{a}$ as follows

$$
\begin{aligned}
b^{a} & =h_{1}\left(\ldots, A, \chi_{\alpha}\right), \\
M & =h_{2}\left(\ldots, A, \chi_{\alpha}\right),
\end{aligned}
$$


where $h_{1}$ and $h_{2}$ are two appropriate functions of the fields involved in the theory. In eq. (8) we underlined the crucial dependence of $h_{1}$ and $h_{2}$ from $A$ and $\chi_{\alpha}$. Now, replacing the solutions (8) in the Lagrangian (7), the degrees of freedom of the Planck multiplet explicitly appear in the matter Lagrangian. Since no Weyl rescaling of the vielbein can remove the auxiliary fields from $\mathscr{L}_{M}$, it follows that the Planck multiplet couples intrinsically to matter. Therefore, there is no way to write the matter Lagrangian as $\mathscr{L}_{M}\left[e_{m}^{a}, \psi_{\alpha}^{a}, \Psi\right]$ by means of a suitable vielbein redefinition of the form $e_{m}^{a} \rightarrow e_{m}^{a} e^{l\left(A, \chi_{\alpha}, F\right)}$, where $l$ is an appropriate function of the components of $\Phi$. In other words, a Jordan frame does not exist for such a theory. The main consequence is that in the MSBD theory the weak equivalence principle is not satisfied by construction and time variations of masses and couplings are not under control. Explicit expressions for eqs. (7) and (8) can be found in [11].

\section{CONCLUSIONS}

In this work we have studied the minimal supersymmetric extension of the BD theory (MSBD) defined by eq. (6). The underlying motivation was the research of possible connections between a Scalar-Tensor interpretation of Dark Energy and a supersymmetric description of Dark Matter. Eq. (6) is obtained replacing the Planck mass with a chiral superfield in the supergravity Lagrangian (4). We called this extra superfield the Planck superfield. Although this approach looks very natural, the resulting phenomenology is radically different from the one of the original BD theory. In the MSBD theory the extra degrees of freedom of the Planck superfield intrinsically couple to matter and a Jordan frame formulation can not be achieved through a suitable vielbein redefinition. As a consequence, this theory does not satisfy the weak equivalence principle by construction. This conclusion could make the minimal supersymmetric extension of the BD idea phenomenologically inconsistent.

\section{REFERENCES}

1. C. Brans and R. H. Dicke, Phys. Rev. 124 (1961) 925.

2. B. Bertotti, L. Iess and P. Tortora, Nature 425 (2003) 374.

3. P. Jordan, Schwerkaft und Weltall (Vieweg, Braunschweig, 1955); M. Fierz, Helv. Phys. Acta 29, 128 (1956); T. Damour and K. Nordtvedt, Phys. Rev. D48, 3436 (1993); T. Damour and A.M. Polyakov, Nucl. Phys. B423, 532 (1994).

4. R. Catena, M. Pietroni and L. Scarabello, Phys. Rev. D 70, 103526 (2004). R. Catena, M. Pietroni and L. Scarabello, [arXiv:astro-ph/0604492].

5. T. Damour, "Gravitation, experiment and cosmology," [arXiv:gr-qc/9606079].

6. N. Bartolo and M. Pietroni, Phys. Rev. D 61 (2000) 023518; G. Esposito-Farese and D. Polarski, Phys. Rev. D 63, 063504 (2001); R. Catena, N. Fornengo, A. Masiero, M. Pietroni and F. Rosati, Phys. Rev. D 70,063519 (2004).

7. E. J. Copeland, M. Sami and S. Tsujikawa, Int. J. Mod. Phys. D 15, 1753 (2006).

8. D. N. Spergel et al. [WMAP Collaboration], Astrophys. J. Suppl. 170, 377 (2007).

9. W. de Boer, "Is dark matter supersymmetric?," Prepared for Cargese School of Particle Physics and Cosmology: the Interface, Cargese, Corsica, France, 4-16 Aug 2003.

10. Julius Wess and Jonathan Bagger, Supersymmetry and Supergravity, Princeton University Press.

11. R. Catena, Phys. Rev. D 75, 044019 (2007). 